\title{
Angular Momentum Coupling and Discrete Quantum Mechanics: the 10-Spin Network, the Pentagonal Relationship, an Eigenvalue Equation and Semiclassical Limits
}

\author{
Robenilson F. dos Santos, Mirco Ragni, Ana Carla P. Bitencourt, \\ Frederico V. Prudente \& Vincenzo Aquilanti
}

\section{Introduction}

Remarkable advances in quantum angular momentum theory and related spin network approaches are of relevance not only to the traditional fields of atomic, molecular and nuclear spectroscopy and dynamics, but also to recent developments in quantum information and quantum relativity [1]. Applications are being developed also for imaging reconstruction, particularly in tomography.

An icosahedral "abacus" representation combines pentagonal and hexagonal relationships. The latter has been recently reconsidered in [2], the pentagonal one is the subject of this work.

Another aspect considered is the relationship with the classification of continuous and discrete polynomials within semiclassical limit illustrated in Figure 1.

\section{Methods}

Recoupling schemes [3,4] can be arranged in pentagonal relationships, allowing to illustrate properties of Wigner $6 \mathrm{j}$ symbols or Racah coefficients [5], Wigner $3 \mathrm{j}$ symbols or Clebsch-Gordan coefficients [6]. Wigner djmm'(b) rotation matrices or symmetrictop wavefunctions. As an important alternative to the generality of treatments, the asymptotic or semiclassical limit connecting $6 \mathrm{j}$ to $3 \mathrm{j}$ and djmm'(b) permits a unifying vision, also to be connected to the modern theory of orthogonal polynomials and the sets of Sturmian orbitals of applied quantum mechanics. Importantly, it generates uniformly the recurrence relationships permitting calculations and asymptotic analysis $[2,5,6]$.

The $6 j$ symbols relate to the $3 \mathrm{j}$ symbols by a semiclassical limit procedure, illustrated graphically by the downward arrow in Fig 1, can be simply written as follows, without specifying the phase and the normalization factor:

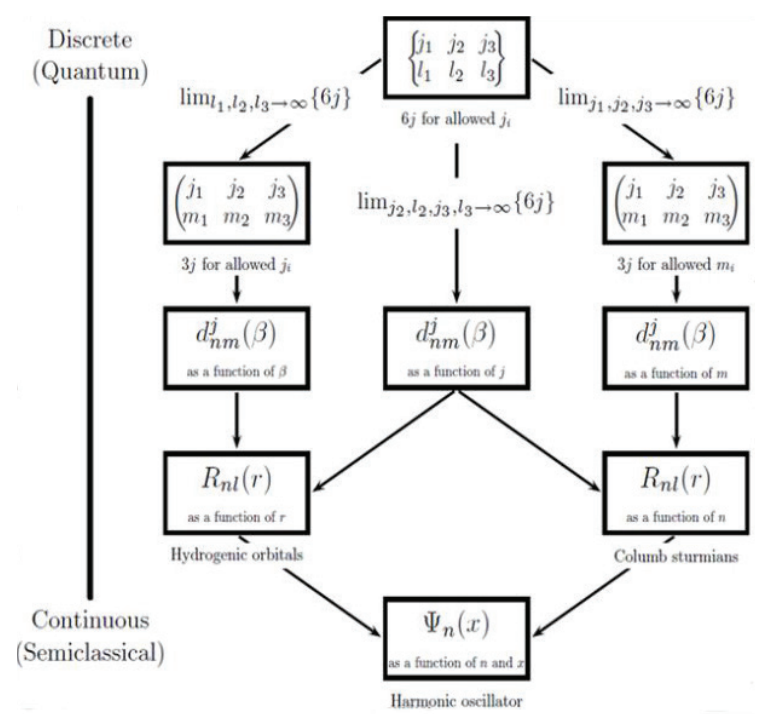

Figure 1. Quantum angular momentum and wavefunctions. 


$$
\left\{\begin{array}{lll}
a & b & x \\
C & D & Y
\end{array}\right\} \longrightarrow\left(\begin{array}{ccc}
a & b & x \\
D-Y & Y-C & C-D
\end{array}\right)
$$

From the viewpoint of interpreting the coupling of two angular momenta, we have the Clebsch-Gordan coefficients relating to the symbols $3 j$ (Figure 2):

$$
\begin{aligned}
& C_{j_{1} j_{2} m_{1} m_{2}}^{j m} \equiv C\left(j_{1} j_{2} j_{3} m_{1} m_{2} m_{3}\right) \\
&\left(\begin{array}{ccc}
j_{1} & j_{2} & j_{3} \\
m_{1} & m_{2} & m_{3}
\end{array}\right)=(-1)^{j_{1}-j_{2}-m_{3}} \frac{1}{\sqrt{2 j_{3}+1}} C_{j_{1} j_{2} m_{1} m_{2}}^{j m} \\
& C_{j_{1} j_{2} m_{1} m_{2}}^{j m}=(-1)^{j_{1}-j_{2}+m_{3}} \sqrt{2 j_{3}+1}\left(\begin{array}{ccc}
j_{1} & j_{2} & j_{3} \\
m_{1} & m_{2} & -m_{3}
\end{array}\right)
\end{aligned}
$$

- $j_{1}, j_{2}$ e $j_{3}$ inteiros ou semi-inteiros não negativos;

- $m_{1}, m_{2}$ e $m_{3}$ inteiros ou semi-inteiros:

- $-j_{1} \leq m_{1} \leq j_{1},-j_{2} \leq m_{2} \leq j_{2}$;

- $\left|j_{1}-j_{2}\right| \leq j \leq j_{1}+j_{2}$;

- $m_{1}+m_{2}=m_{3}$.

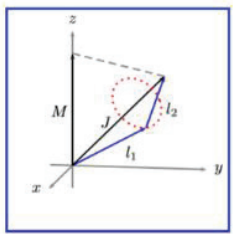

Figure 2. Illustration of the coupling of two angular momenta giving a third one and of the associated projections, as they occur in the ClebschGordan coefficient or equivalently in Wigner $3 \mathrm{j}$ symbols.

\section{Results and discussion}

\section{THE PENTAGONAL RELATIONSHIP}

Making use of the tree structures, several relationships between 3nj symbols can be graph. If we consider relationships involving only different tree structures, i.e. without any permutation in the order of the labels a, b, c and d, so that only recoupling operations are considered, we obtain the pentagonal scheme. Figure 3 illustrates graphically the origin of the pentagonal relationships in angular momentum theory known as the BiedenharnElliot identity, that we write in a rearranged form as follows and illustrate in Fig 3:

$$
\begin{gathered}
\left\{\begin{array}{lll}
j_{a} & j_{b} & j_{a b} \\
j_{c} & j_{a b c} & j_{b c}
\end{array}\right\}= \\
\sum_{j_{d a j} j_{c c d}}(-)^{x}\left(2 j_{a b c}+1\right)\left(2 j_{c d}+1\right)\left(2 j_{b c d}+1\right) \\
\left\{\begin{array}{ccc}
j_{a} & j_{b c} & j_{a b c} \\
j_{d} & j & j_{b c d}
\end{array}\right\}\left\{\begin{array}{ccc}
j_{b} & j_{c} & j_{b c} \\
j_{d} & j_{b c d} & j_{c d}
\end{array}\right\} \\
\left\{\begin{array}{ccc}
j_{a} & j_{b} & j_{a b} \\
j_{c d} & j & j_{c d}
\end{array}\right\}\left\{\begin{array}{lll}
j_{a b} & j_{c} & j_{a b c} \\
j_{d} & j & j_{c d}
\end{array}\right\} \\
x=j_{a}+j_{b}+j_{c}+j_{a b}+j_{b c}+j_{b c d}+j_{c d}-j_{a b c}-j_{d}-j
\end{gathered}
$$
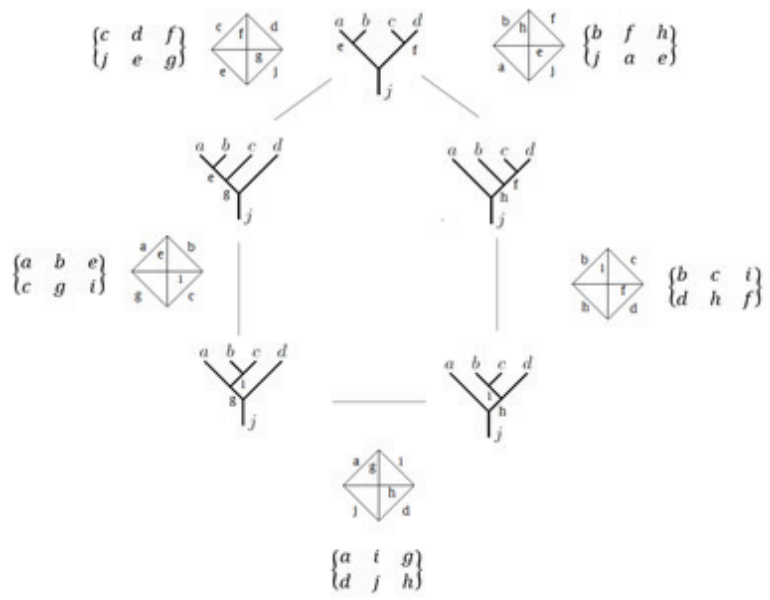

Figure 3. Illustration of the pentagonal relationship, exhibiting the coupling scheme trees, their representation as tetrahedra and the associated $6 \mathrm{j}$ symbols.

\section{RECURRENCE RELATIONSHIPS AS EIGENVALUE EQUATIONS}

The three-term relationship for $6 j$ symbols can be obtained by specializing to specific values entries in the above equations and imposing increase of one of the angular momenta in unity steps. A way of writing here, useful for computation and semiclassical analysis, is:

$j_{b c} E\left(j_{b c}+1\right)\left\{\begin{array}{ccc}j_{a} & j_{b} & j_{a b} \\ j_{c} & j & j_{b c}+1\end{array}\right\}+F\left(j_{b c}\right)\left\{\begin{array}{ccc}j_{a} & j_{b} & j_{a b} \\ j_{c} & j & j_{b c}\end{array}\right\}+\left(j_{b c}+1\right) E\left(j_{b c}\right)\left\{\begin{array}{ccc}j_{a} & j_{b} & j_{a b} \\ j_{c} & j & j_{b c}-1\end{array}\right\}=0$

where

$$
\begin{aligned}
E\left(j_{b c}\right)=\{ & {\left.\left[j_{b c}^{2}-\left(j-j_{a}\right)^{2}\right]\left[\left(j+j_{a}+1\right)^{2}-j_{b c}^{2}\right]\left[j_{b c}^{2}-\left(j_{c}-j_{b}\right)^{2}\left[\left(j_{b}+j_{c}+1\right)^{2}-j_{b c}^{2}\right]\right]\right\}^{1 / 2} } \\
F\left(j_{b c}\right)=\left(2 j_{b c}+1\right)\left\{j_{b c}\left(j_{b c}+1\right)\left[-j_{b c}\left(j_{b c}+1\right)+j_{b}\left(j_{b}+1\right)+j_{c}\left(j_{c}+1\right)\right]\right. & +j_{j}\left(j_{a}+1\right)\left[j_{b c}\left(j_{b c}+1\right)-j_{b}\left(j_{b}+1\right)+j_{c}\left(j_{c}+1\right)\right] \\
& \left.+j(j+1)\left[j_{b c}\left(j_{b c}+1\right)+j_{b}\left(j_{b}+1\right)-j_{c}\left(j_{c}+1\right)\right]-2 j_{b c}\left(j_{b c}+1\right) j_{a b}\left(j_{a b}+1\right)\right\}
\end{aligned}
$$


When rearranged as an eigenvalue equation, this relationship can be interpreted as a second order finite difference equation, and therefore as a discrete Schrödinger equation of interest for applied quantum mechanics.

\section{QUANTUM AND SEMICLASSICAL SPIN NETWORKS:}

Spin networks are illustrated here for the case of two quantum systems, as described by coupling schemes associated to tree-like structures, each endowed with angular momenta, which can assume in usual cases integer or half-integer values in terms of reduced Planck's constant. In practice, they actually can be not only an electronic or nuclear spin but also e.g. rotational and orbital, giving a state with total angular momentum $\mathrm{j}$ (coupling described by e.g. spin-orbit terms in the Hamiltonian).

Figure 4 illustrates schematically the combination of pentagonal and hexagonal relationships of these angular momenta. Representations of couplings as trees extend those in Fig 3 to an additional angular momentum and permit the introduction of the $9 j$ coupling scheme as a combination of intermediate coupling along a path on the abacus.

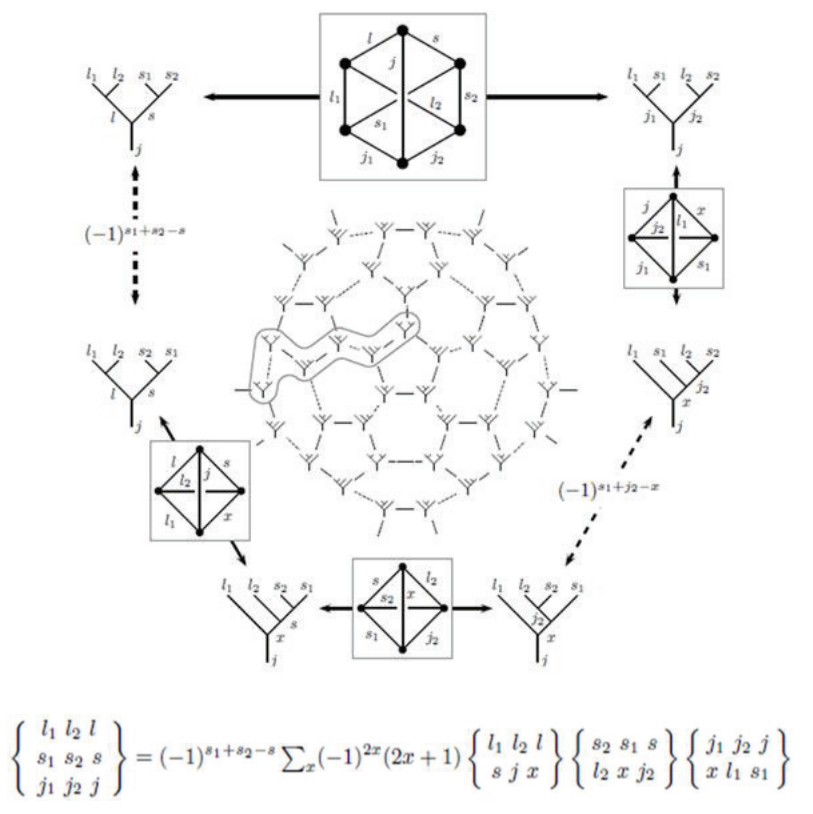

Figure 4. Icosahedral "abacus" representation, combining the hexagonal relationship rediscussed in Ref 2, and the pentagonal relationship reconsidered in this work.

\section{Conclusions}

Numerical exact and approximate calculations can be explicitly carried out following the techniques developed previously [7]. Besides general use in the contexts mentioned, the results are of perspective use in solutions of quantum mechanical problems by discretization algorithms. In general relativity, spacetime is discretized by Regge calculus; spin-networks originated from PonzanoRegge asymptotics; Regge symmetries are crucial for our developments: we dedicate this modest contribution to the memory of Professor Tullio Regge (Torino, 1931-2014).

\section{Acknowledgments}

The authors are grateful for the support given from the CAPES and CNPQ. Also, V.A. thanks CAPES for a PVE position at UFBA.

\section{References}

1. V. Aquilanti, A.C.P. Bitencourt, C. da S. Ferreira, A. Marzuoli, M. Ragni, Quantum and semiclassical spin networks: from atomic and molecular physics to quantum computing and gravity, Physica Scripta, 2008, 78, 058103.

2. D. Marinelli, A. Marzuoli, V. Aquilanti, R. Anderson, A.C.P. Bitencourt, M. Ragni, Symmetric Angular Momentum Coupling, the Quantum Volume Operator and the 7-spin Network: A Computational Perspective, Lecture Notes in Computer Science, 2014, 8579,508

3. V. Aquilanti, G. Grossi, Angular momentum coupling schemes in the quantum mechanical treatment of P-state atom collisions, J. Chem. Phys. 1980, 73, 11654.

4. V. Aquilanti, S. Cavalli, G. Grossi, Hund's cases for rotating diatomic molecules and atomic collisions: angular momentum couplings and orbital alignment, Z. Phys. D. 1996, 36, 215.

5. R.W. Anderson, V. Aquilanti, A.C.P. Bitencourt, D. Marinelli, M. Ragni, The Screen Representation of Spin Networks: 2D Recurrence, Eigenvalue Equation for 6j Symbols, Geometric Interpretation and Hamiltonian Dynamics, Lecture Notes in Computer Science. 2013, 7972, 46.

6. A.C.P. Bitencourt, M. Ragni, R.G. Littlejohn, R. Anderson, V. Aquilanti, The Screen Representation of Vector Coupling Coefficients or Wigner 3j Symbols: Exact Computation and Illustration of the Asymptotic Behavior, Lecture Notes in Computer Science. 2014, 8579, 468.

7. M. Ragni, A.C.P. Bitencourt, C.S. Ferreira, V. Aquilanti, R.W. Anderson, R.G. Littlejohn, Exact computation and asymptotic approximations of $6 \mathrm{j}$ symbols: Illustration of their semiclassical limits, Int. J. Q. Chem. 2010, 110, 731 


\title{
Robenilson F. dos Santos ${ }^{1,2^{*}}$, \\ Mirco Ragni ${ }^{3}$, Ana Carla \\ P. Bitencourt ${ }^{3}$, Frederico \\ V. Prudentel \& Vincenzo \\ Aquilanti ${ }^{1,4}$
}

\author{
${ }^{1}$ Instituto de Física, Universidade Federal da Bahia, Brasil; \\ ${ }^{2}$ Instituto Federal de Alagoas, Campus Piranhas, Brasil; \\ ${ }^{3}$ Departamento de Física, Universidade Estadual de Feira de Santana, \\ Brasil; \\ ${ }^{4}$ Departamento de Química, Biologia e Biotecnologia, Universidade de \\ Perugia, Itália \\ *E-mail: roferreirafs@gmail.com
}

